Case Study

\title{
The effect of white matter integrity on functional outcome in central pontine demyelination
}

\author{
Kyoung Bo Lee, PT, PhD ${ }^{1)}$, Bo Young Hong, MD, PhD ${ }^{1)}$, Joon Sung Kim, MD, $\mathrm{PhD}^{1)}$, \\ Dong Back Son, PT, MPT ${ }^{1)}$, SAng Il Choi, $\mathrm{PT}^{1)}$, Seong Hoon Lim, MD, $\mathrm{PhD}^{1)^{*}}$
}

1) Department of Rehabilitation Medicine, St. Vincent's Hospital, College of Medicine, The Catholic University of Korea: 222 Banpo-daero, Seocho-gu, Seoul, Republic of Korea

\begin{abstract}
Purpose] Central pontine demyelination is a common osmotic demyelination syndrome. Typically, central pontine demyelination shows high signal intensity with demyelination in the mid-pons. Despite severe imaging findings, patients usually recover. This study investigated the integrity of the corticospinal tract (CST) and corticoreticular pathway (CRP) in patients at 12 months after the onset of central pontine demyelination. [Participants and Methods] This retrospective cross-sectional observational study assessed two patients with central pontine demyelination, who were ultimately able to walk without using an aid or orthosis and to use both hands but with mild residual symptoms. All participants underwent diffusion tensor imaging (DTI) at 12 months after onset. [Results] Both patients had mild ataxia at 12 months after onset. The integrity of the CST was restored in both cases, while the integrity of the CRP recovered partially. [Conclusion] The integrity of the CST and CRP at 12 months after the onset of central pontine demyelination was similar in both cases as well as the recovery status of the hand function and ambulation. These results may be useful in planning therapy for patients with central pontine demyelination.

Key words: Central pontine demyelination, Diffusion tensor imaging, Prognosis
\end{abstract}

(This article was submitted Mar. 14, 2019, and was accepted May 15, 2019)

\section{INTRODUCTION}

Osmotic demyelination syndromes include central pontine and extrapontine myelinolysis ${ }^{1)}$. In these syndromes, central pontine demyelination involves symmetrical myelin sheath disruption in areas where the white matter becomes exposed to osmotically active substances cleared from edematous grey matter, namely, the crossing pontocerebellar fibers within the pontine nuclei and other grey matter regions containing heavily myelinated fibers, such as the cerebellum and lateral geniculate body ${ }^{2}$. Clinical recovery from osmotic pontine demyelination can occur with correction of the electrolyte imbalance and underlying systemic disease ${ }^{2-4}$. More than half of patients with osmotic pontine demyelination have favorable outcomes ${ }^{1)}$. The radiological findings of central pontine demyelination vary and include cortical and subcortical types ${ }^{4,5)}$. However, the integrity of the white matter in central pontine demyelination is not known.

This case report describes two patients with central pontine demyelination who had severe quadriplegia and underwent rehabilitation; in the chronic stage, they both had mild ataxia.

\section{PARTICIPANTS AND METHODS}

This was a retrospective cross-sectional observational clinical trial. Two right-handed patients with osmotic central pontine demyelination were recruited from the Department of Rehabilitation Medicine, St. Vincent's Hospital, Suwon, South Korea

*Corresponding author. Seong Hoon Lim (E-mail: seonghoon@catholic.ac.kr)

(C2019 The Society of Physical Therapy Science. Published by IPEC Inc.

(c) (i) $\odot$ This is an open-access article distributed under the terms of the Creative Commons Attribution Non-Commercial No DerivaCC BY NC ND tives (by-nc-nd) License. (CC-BY-NC-ND 4.0: https://creativecommons.org/licenses/by-nc-nd/4.0/) 
between January 2015 and December 2018. Considering the maturation of neurological recovery, completion of neurological recovery was defined as 6 months after onset ${ }^{6}$.

Demographic, clinical, and brain magnetic resonance imaging (MRI) data were obtained for both participants. Brain MRI diffusion tensor imaging (DTI) was performed around 12 months after onset, at the time of a functional evaluation. All participants received routine physical and occupational therapy for 1-2 hours per day, 5 days per week. The study protocol was reviewed and approved by the Institutional Review Board of The Catholic University, College of Medicine (Registry No. VC17ZESE0152); the board waived the need for informed consent.

The DTI was performed using a 3.0-T MRI (MAGNETOM ${ }^{\circledR}$ Verio, Siemens, Erlangen, Germany) equipped with a sixchannel head coil. The data were acquired in the form of single-shot spin-echo echo-planar images, with axial slices covering the entire brain across 76 2.0-mm-thick interleaved slices (no gaps): repetition time (TR)/echo time (TE) $=14,300 / 84 \mathrm{~ms}$; field of view=224 $\times 224 \mathrm{~mm}^{2}$; matrix $224 \times 224$; voxel size $1 \times 1 \times 2 \mathrm{~mm}^{3}$; number of excitations=1. Diffusion-sensitizing gradients were applied in 64 non-collinear directions with a b-value of $1,000 \mathrm{~ms} / \mathrm{mm}^{2}$. The $b=0$ images were scanned before acquiring the diffusion-weighted images ( 65 volumes in total $)^{7}$.

DTI-Studio software (CMRM, Johns Hopkins Medical Institute, MD, USA) was used to reconstruct the corticospinal tract (CST) and corticoreticular pathway (CRP). Fiber tracking was based on fiber assignment continuous tracking (FACT) algorithm and a multiple regions of interest (ROIs) approach using DTI-Studio ${ }^{8,9}$ ). ROIs were used for CST and CRT reconstruction. The initial ROI was placed on the pontomedullary junction portion of the CST, and the second on the anterior mid-pons portion of the CST (Fig. 1) ${ }^{10}$ ). To analyze the CRP, the seed ROI was placed on the reticular formation of the medulla and the target ROI on the midbrain tegmentum ${ }^{11)}$. The termination criteria were fractional anisotropy $(\mathrm{FA})>0.2$ and an angle change of $<60$ degrees $^{12)}$.

\section{RESULTS}

A 40-year-old man (Case 1) with diabetes mellitus, hypertension, and chronic alcoholic hepatitis presented to the emergency room with sudden memory impairment with no trauma. He was treated for hepatic encephalopathy and hyponatremia (serum sodium $105 \mathrm{mEq} / \mathrm{L}$ ). While correcting the sodium (daily 2-7 mEq/L), he became increasingly drowsy. A 57-year-old man (Case 2) with poor oral intake, hypertension, and chronic alcoholism presented to the emergency room with generalized weakness of all limbs with no trauma. He was treated for hyponatremia (serum sodium $107 \mathrm{mEq} / \mathrm{L}$ ), anemia, and thrombocytopenia in the Department of Internal Medicine. After the acute treatment, quadriplegia was noted. Their finding of MRI showed central pontine demyelination. In the early stage, on manual muscle testing (MMT), they had proximal limb weakness greater than distal weakness, graded poor and fair (Case 1) and graded poor+ and fair+ (Case 2) respectively. They had no restrictions in range of motion (ROM). Main problems of those were postural instability due to trunk and proximal muscle weakness and ataxic movements.

They got rehabilitation programs including physical therapy and occupational therapy, 30 min per session, 2-3 sessions a day, five times a week. In particular, physiotherapy focused on core stabilization, proximal gross muscle strengthening, and trunk and limb coordination training. They underwent sit-to-stand, assisted-standing, and sitting and standing balance training. They started gait training with moderate assistance at 5 weeks after onset and progressed to gait training with supervised use of sand bags on both ankles. Case 1 underwent body-weight-supported treadmill training (BWSTT) for 30 minutes, beginning with a $40 \%$ decrease in weight to full weight acceptance, and with an average speed ranging from 0.3 to $0.5 \mathrm{~m} / \mathrm{s}$. After rehabilitation for 12 weeks, he ambulated without a walking aid or orthosis. Case 2 started BWSTT for 20 minutes beginning with a $40 \%$ decrease in weight to full weight acceptance, with average speed ranging from 0.2 to $0.4 \mathrm{~m} / \mathrm{s}$. After rehabilitation for 20 weeks, he ambulated with a cane, without an orthosis. One year after onset, they had regained most of his functional abilities for gait and hand function. However, they still complained of ataxia and sensory changes in the lower limbs. Follow-up MRI showed large central high signal intensities in the pons. The white matter integrity evaluated by DTI showed a relatively well-preserved CST compared with the CRP (Figs. 1 and 2).

\section{DISCUSSION}

Studies have demonstrated the clinical usefulness of DTI for evaluating function in two patients with central pontine demyelination. The integrity of the white matter, i.e., the CST and CRP, in central pontine demyelination may be an important factor of good functional recovery. The relationship between DTI CST images and hand function has been evaluated several times $^{8,13-16)}$. Most reports have shown that damage to the CST is useful for predicting the restoration of motor function in patients with stroke ${ }^{13,16-18)}$. Consistent with previous studies, the CST integrity reflected the functional status of the central pontine demyelination in our patients.

Central pontine demyelination follows a biphasic course with initial seizures or encephalopathy that may improve gradually, but which are then followed by severe deterioration manifesting as dysarthria, dysphagia, oculomotor dysfunction, and variable degrees of quadriparesis ${ }^{1}$. The typical radiological findings on brain MRI are hyperintense lesions in the central pons or associated extrapontine structures on T2-weighted and fluid-attenuated inversion recovery sequences, with corresponding hypointensity on $\mathrm{T} 1$-weighted sequences ${ }^{1,4,5)}$. It is very difficult to predict the prognosis of central pontine 


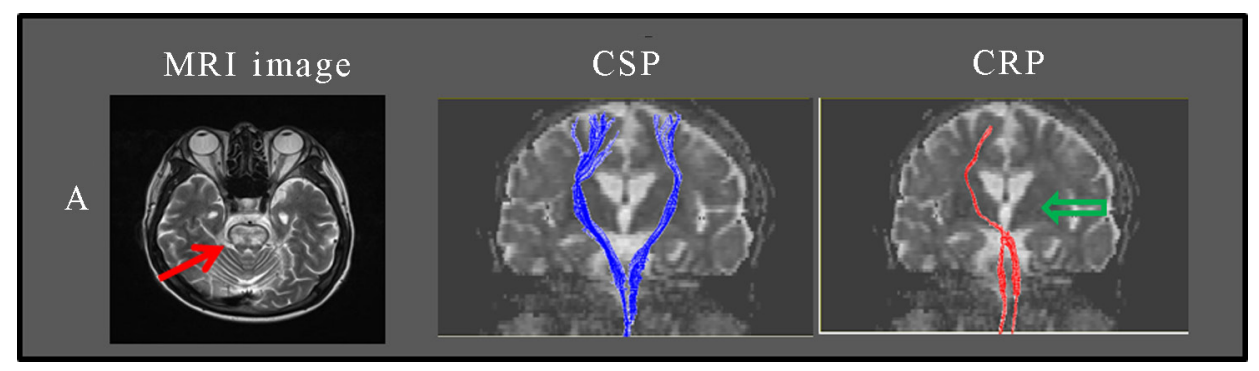

Fig. 1. Diffusion tensor tractography of the corticospinal tract (CST) and corticoreticular pathway (CRP). A: CASE \#1, in MRI T2 scan, red arrow indicates brain lesion. The integrity of the CST was preserved from the cortex to the pons at the brainstem. However, the CRP was discontinuous (green arrow) at the left subcortical white matter and brainstem.

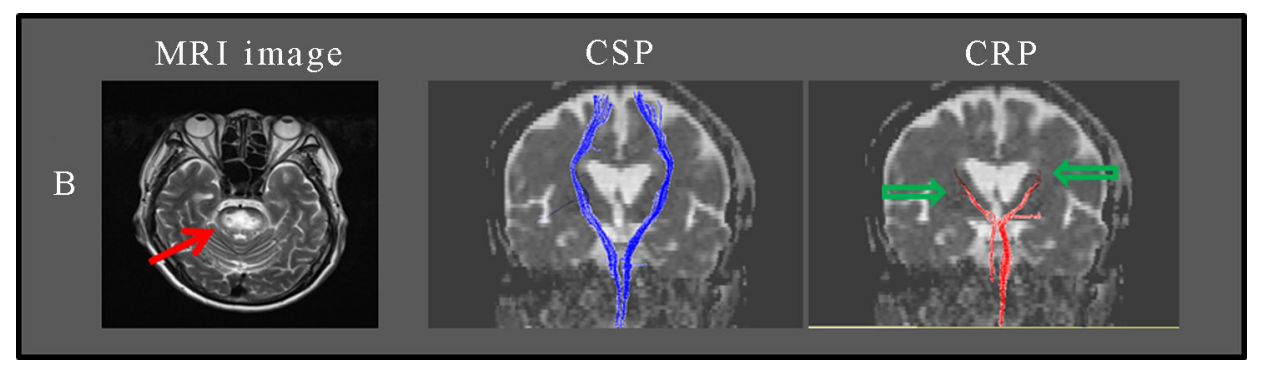

Fig. 2. Diffusion tensor tractography of the corticospinal tract (CST) and corticoreticular pathway (CRP). B: CASE \#2, in MRI T2 scan, red arrow indicates brain lesion. The integrity of the CST was preserved from the cortex to the pons at the brainstem. However, the CRP was discontinuous (green arrows) at the subcortical white matter and brainstem on the both sides.

demyelination. Conventional MRI does not match the clinical findings. Therefore, we used DTI to predict the outcome and explain the central pontine demyelination.

The CRP is an extrapyramidal motor pathway that innervates the proximal muscles of the extremities and the axial muscles; it plays roles in postural control and locomotor function ${ }^{19}$. The CRP originates mainly from the premotor cortex $(\mathrm{PMC})^{19}$. Associations of the PMC lesions with proximal muscle weakness ${ }^{20)}$, gait recovery ${ }^{21,22)}$, and postural control have been reported ${ }^{21)}$. Both of our patients had proximal weakness and balance problems during rehabilitation.

The CST integrity is reflected in the recovery of upper limb motor function ${ }^{13-16)}$, while the CRP integrity is reflected in the recovery of lower limb motor function ${ }^{19,23)}$. Both of our patients with central pontine demyelination fully recovered their hand function, while lower limb ataxia remained. These findings are consistent with previous studies. However, our patients' problems cannot be fully explained using only the CST and CRP findings. In early DTI images, they had normal CST integrity, but disconnection of the CRP; it is possible that the CST takes over CRP functions during recovery.

A few limitations of our study should be noted. First, we used a cross-sectional design; we did not collect tensor images in the early stage, so the changes in DTI over time were not investigated. Second, we evaluated only two patients because of the rarity of central pontine demyelination. To overcome this methodological limitation, further larger-scale study is needed to confirm the role of DTI in patients with central pontine demyelination.

In conclusion, the integrity of the CST and CRP was consistent with clinical recovery in patients with central pontine demyelination. These results will be useful for predicting functional restoration of the hand and gait in the acute phase in patients with central pontine demyelination, and will contribute to our understanding of the functional prognosis of central pontine demyelination.

Conflicts of interest

The authors declare no financial or other conflicts of interest.

\section{ACKNOWLEDGMENT}

This research was supported by Basic Science Research Program through the National Research Foundation of Korea (NRF) funded by the Ministry of Science and ICT (grant number: 2017R1E1A1A01074324). 


\section{REFERENCES}

1) Singh TD, Fugate JE, Rabinstein AA: Central pontine and extrapontine myelinolysis: a systematic review. Eur J Neurol, 2014, 21: 1443-1450. [Medline] [CrossRef]

2) Varanda S, Costa S, Carvalho R, et al.: Central pontine myelinolysis caused by hypernatremia. J Neurol Sci, 2016, 370: 274-276. [Medline] [CrossRef]

3) Alleman AM: Osmotic demyelination syndrome: central pontine myelinolysis and extrapontine myelinolysis. Semin Ultrasound CT MR, 2014, 35: 153-159. [Medline] [CrossRef]

4) Kumar S, Fowler M, Gonzalez-Toledo E, et al.: Central pontine myelinolysis, an update. Neurol Res, 2006, 28: 360-366. [Medline] [CrossRef]

5) Tatewaki Y, Kato K, Tanabe Y, et al.: MRI findings of corticosubcortical lesions in osmotic myelinolysis: report of two cases. Br J Radiol, 2012, 85: e87-e90. [Medline] [CrossRef]

6) Lee KB, Kim JS, Hong BY, et al.: The motor recovery related with brain lesion in patients with intracranial hemorrhage. Behav Neurol, 2015, 2015: 258161. [Medline] [CrossRef]

7) Kim Y, Kim SH, Kim JS, et al.: Modification of cerebellar afferent pathway in the subacute phase of stroke. J Stroke Cerebrovasc Dis, 2018, 27: 2445-2452. [Medline] [CrossRef]

8) Jang SH, Kim K, Kim SH, et al.: The relation between motor function of stroke patients and diffusion tensor imaging findings for the corticospinal tract. Neurosci Lett, 2014, 572: 1-6. [Medline] [CrossRef]

9) Jiang H, van Zijl PC, Kim J, et al.: DtiStudio: resource program for diffusion tensor computation and fiber bundle tracking. Comput Methods Programs Biomed, 2006, 81: 106-116. [Medline] [CrossRef]

10) Yeo SS, Choi BY, Chang CH, et al.: Periventricular white matter injury by primary intraventricular hemorrhage: a diffusion tensor imaging study. Eur Neurol, 2011, 66: 235-241. [Medline] [CrossRef]

11) Yeo SS, Chang MC, Kwon YH, et al.: Corticoreticular pathway in the human brain: diffusion tensor tractography study. Neurosci Lett, 2012, 508: 9-12. [Medline] [CrossRef]

12) Jang SH, Seo JP: The distribution of the cortical origin of the corticoreticular pathway in the human brain: a diffusion tensor imaging study. Somatosens Mot Res, 2014, 31: 204-208. [Medline] [CrossRef]

13) Cho SH, Kim DG, Kim DS, et al.: Motor outcome according to the integrity of the corticospinal tract determined by diffusion tensor tractography in the early stage of corona radiata infarct. Neurosci Lett, 2007, 426: 123-127. [Medline] [CrossRef]

14) Groisser BN, Copen WA, Singhal AB, et al.: Corticospinal tract diffusion abnormalities early after stroke predict motor outcome. Neurorehabil Neural Repair, 2014, 28: 751-760. [Medline] [CrossRef]

15) Kim AR, Kim DH, Park SY, et al.: Can the integrity of the corticospinal tract predict the long-term motor outcome in poststroke hemiplegic patients? Neuroreport, 2018, 29: 453-458. [Medline] [CrossRef]

16) Rosso C, Valabregue R, Attal Y, et al.: Contribution of corticospinal tract and functional connectivity in hand motor impairment after stroke. PLoS One, 2013, 8: e73164. [Medline] [CrossRef]

17) Yoo YJ, Kim JW, Kim JS, et al.: Corticospinal tract integrity and long-term hand function prognosis in patients with stroke. Front Neurol, 2019, 10: 374. [Medline] [CrossRef]

18) Seo JP, Do KH, Jung GS, et al.: The difference of gait pattern according to the state of the corticospinal tract in chronic hemiparetic stroke patients. NeuroRehabilitation, 2014, 34: 259-266. [Medline]

19) Matsuyama K, Mori F, Nakajima K, et al.: Locomotor role of the corticoreticular-reticulospinal-spinal interneuronal system. Brain mechanisms for the integration of posture and movement2004, pp 239-249.

20) Freund H-J, Hummelsheim H: Premotor cortex in man: evidence for innervation of proximal limb muscles. Exp Brain Res, 1984, 53: 479-482. [Medline]

21) Chang WH, Tang PF, Wang YH, et al.: Role of the premotor cortex in leg selection and anticipatory postural adjustments associated with a rapid stepping task in patients with stroke. Gait Posture, 2010, 32: 487-493. [Medline] [CrossRef]

22) Miyai I, Yagura H, Hatakenaka M, et al.: Longitudinal optical imaging study for locomotor recovery after stroke. Stroke, 2003, 34: 2866-2870. [Medline] [CrossRef]

23) Jang SH, Chang CH, Lee J, et al.: Functional role of the corticoreticular pathway in chronic stroke patients. Stroke, 2013, 44: 1099-1104. [Medline] [CrossRef] 